\title{
$\beta$-Hydroxybutyrate Detection with Proton MR Spectroscopy in Children with Drug-Resistant Epilepsy on the Ketogenic Diet
}

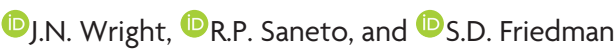

\begin{abstract}
BACKGROUND AND PURPOSE: The ketogenic diet, including both classic and modified forms, is an alternative to antiepileptic medications used in the treatment of drug-resistant epilepsy. We sought to evaluate the utility of proton MR spectroscopy for the detection of $\beta$-hydroxybutyrate in a cohort of children with epilepsy treated with the ketogenic diet and to correlate brain parenchymal metabolite ratios obtained from spectroscopy with $\beta$-hydroxybutyrate serum concentrations.
\end{abstract}

MATERIALS AND METHODS: Twenty-three spectroscopic datasets acquired at a TE of $288 \mathrm{~ms}$ in children on the ketogenic diet were analyzed with LCModel using a modified basis set that included a simulated $\beta$-hydroxybutyrate resonance. Brain parenchymal metabolite ratios were calculated. Metabolite ratios were compared with serum $\beta$-hydroxybutyrate concentrations, and partial correlation coefficients were calculated using patient age as a covariate.

RESULTS: $\beta$-hydroxybutyrate blood levels were highly correlated to brain $\beta$-hydroxybutyrate levels, referenced as either choline, creatine, or $\mathrm{N}$-acetylaspartate. They were inversely but more weakly associated with $\mathrm{N}$-acetylaspartate, regardless of the ratio denominator. No strong concordance with lactate was demonstrated.

CONCLUSIONS: Clinical MR spectroscopy in pediatric patients on the ketogenic diet demonstrated measurable $\beta$-hydroxybutyrate, with a strong correlation to $\beta$-hydroxybutyrate blood levels. These findings may serve as an effective tool for noninvasive monitoring of ketosis in this population. An inverse correlation between serum $\beta$-hydroxybutyrate levels and brain tissue $N$-acetylaspartate suggests that altered amino acid handling contributes to the antiepileptogenic effect of the ketogenic diet.

ABBREVIATIONS: $\mathrm{BHB}=\beta$-hydroxybutyrate; $\mathrm{GABA}=$ gamma-aminobutyric acid; $\mathrm{KD}=$ ketogenic diet; $\mathrm{MAD}=$ modified Atkins diet

T he ketogenic diet (KD) is a high-fat, low-carbohydrate, low-protein diet developed in the 1920 s as a treatment for epilepsy, in response to the observation that fasting decreased the incidence and severity of seizures. ${ }^{1}$ Use of the KD was largely supplanted by the emergence of multiple highly effective antiepileptic medications throughout the subsequent decades. ${ }^{2}$ More recently, there has been a resurgence of interest in the KD, especially for treatment of drug-resistant epilepsy in children. Clinical research has demonstrated that modified forms of the diet, including the modified Atkins diet (MAD)

Received November 6, 2017; accepted after revision March 5, 2018.

From the Department of Radiology (J.N.W., S.D.F.) and Department of Neurology, Division of Pediatric Neurology (R.P.S.), University of Washington and Seattle Children's Hospital, Seattle, Washington.

Please address correspondence to Jason N. Wright, MD, Department of Radiology, M/S MA.7.220, Seattle Children's Hospital, PO Box 5371, Seattle, WA 98145; e-mail: jnixon@uw.edu

http://dx.doi.org/10.3174/ajnr.A5648 and modified ratio formulations of the $\mathrm{KD}$, are also effective in controlling seizures. ${ }^{3}$

The KD restricts intake of carbohydrates and protein, driving the production of ketone bodies from fat by the liver. This alteration in energetic substrates results in increased serum levels of $\beta$-hydroxybutyrate (BHB) and, to a lesser degree, acetone and acetoacetate. Brain parenchymal concentrations of ketone bodies have been shown to increase as a function of serum concentrations, ${ }^{4}$ modulated by the duration of hyperketonemia. Prolonged ketosis results in upregulation of the monocarboxylic acid transporter family of proteins that governs the active transport of ketone bodies from serum, resulting in increased steady-state brain tissue concentrations. ${ }^{5}$ Ketone bodies serve as the primary oxidative substrate for neurons during ketosis. ${ }^{6}$

The mechanisms by which the KD exerts its antiepileptogenic effect are not definitely known. Theories that have been advanced include the following: an anticonvulsant effect of ketone bodies; a shift in brain amino acid handling favoring increased synthesis of the inhibitory neurotransmitter gamma- 

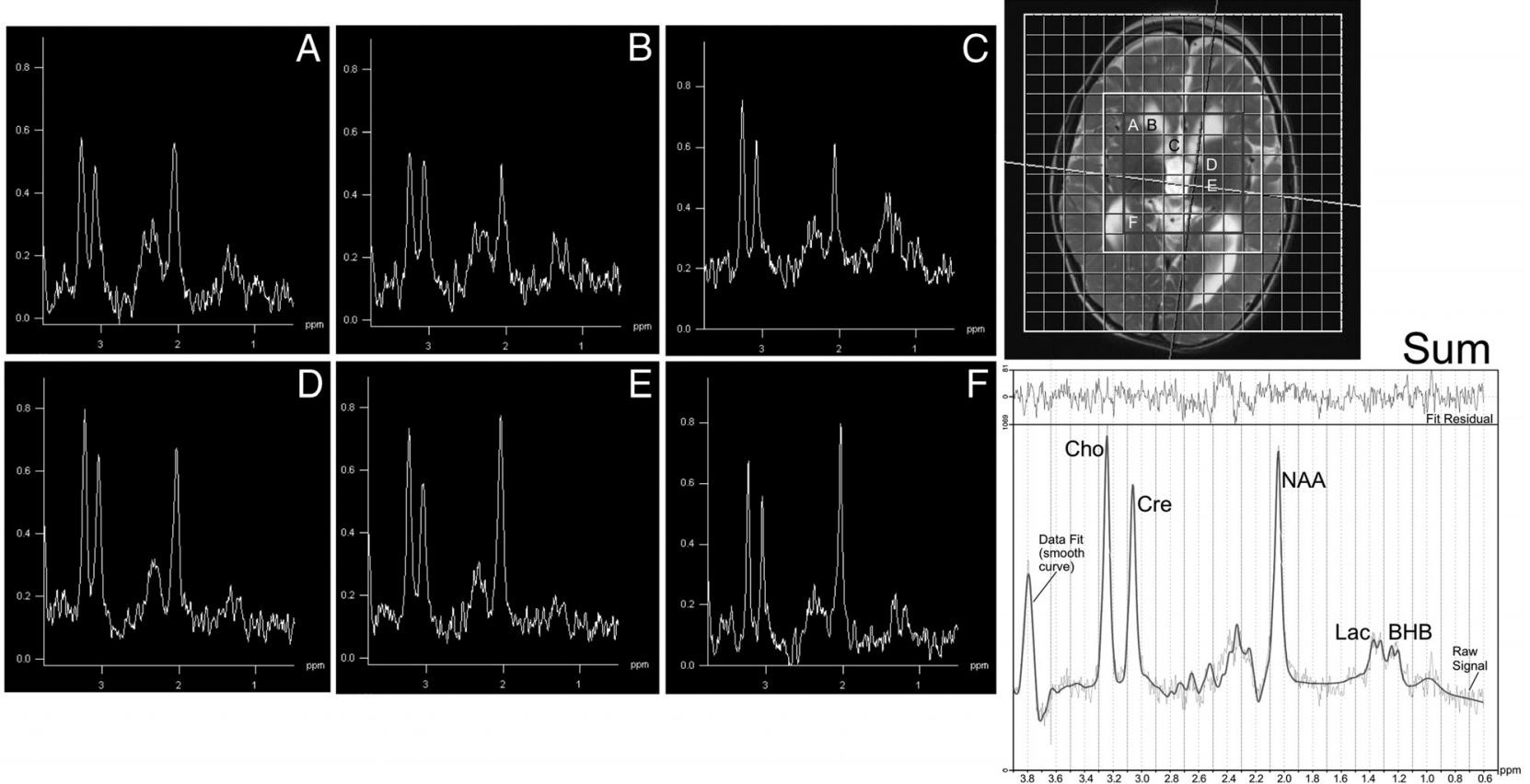

FIG 1. Voxel-of-interest (inner $8 \times 8$ matrix) and masked region $(7 \times 7$ matrix) that retained spectra based on SNR, NAA, and adequate Cramér-Rao bounds. Across the brain (spectra labeled $A-F$, corresponding to labeled voxels in image at the upper right), 2 peaks comprising lactate and BHB are shown at 1.33 and $1.2 \mathrm{ppm}$, respectively, with lactate more prominent in voxels containing CSF in this patient with mitochondrial cytopathy. At the lower right, the composite spectrum from the masked region is shown, with the LCModel fit overlaid.

aminobutyric acid (GABA); a direct consequence of glucose restriction; or an effect mediated by altered polyunsaturated fatty acid metabolism. ${ }^{7}$ Whatever the mechanism, steady-state blood levels of BHB have been shown to correlate with the degree of seizure control. ${ }^{8}$

Proton MR spectroscopy ( $\left({ }^{1} \mathrm{H}-\mathrm{MR}\right.$ spectroscopy) is a clinically available imaging technique by which tissue concentrations of metabolites can be noninvasively evaluated. Anecdotally, we have observed a resonance near $1.2 \mathrm{ppm}$ on long-TE $(288 \mathrm{~ms})$ spectra in patients treated with the $\mathrm{KD}$, tentatively attributed to $\mathrm{BHB}$. There have been rare anecdotal reports of BHB detection by ${ }^{1} \mathrm{H}-\mathrm{MR}$ spectroscopy in the brain parenchyma of children treated with the $\mathrm{KD},{ }^{9,10}$ with only 4 patients reported to date. We sought to evaluate the utility of ${ }^{1} \mathrm{H}-\mathrm{MR}$ spectroscopy for the routine detection of $\mathrm{BHB}$ in a cohort of children treated with the KD for drug-resistant epilepsy and to correlate ${ }^{1} \mathrm{H}-\mathrm{MR}$ spectroscopy metabolite ratios with $\mathrm{BHB}$ serum concentrations.

\section{MATERIALS AND METHODS}

Following institutional review board approval, we retrospectively identified 247 patients treated with the classic KD, a modifiedratio KD, or the MAD at our institution between 2006 and 2014. The classic KD used a ratio of 4:1, indicating a target dietary intake of $4 \mathrm{~g}$ of fat per gram of combined carbohydrate and protein. Patients were shifted to a modified-ratio formulation of the KD depending on clinical course and seizure response. The MAD, which limits carbohydrate intake to a maximum of $20 \mathrm{~g}$ per day, was used in some patients to increase tolerability and compliance.

Of the 247 patients, 38 underwent $\geq 1{ }^{1} \mathrm{H}$-MR spectroscopy during their treatment, for a total of $49 \mathrm{MR}$ spectroscopy datasets. Clinical data on patient age, epilepsy and seizure type, diet type, medications, serum BHB concentration, and lag in days between blood testing and MR spectroscopy were recorded after retrospective chart review.

Data were further reduced to 23 spectra from 18 total patients in whom the lag between blood BHB sampling and MR spectroscopy was $<30$ days. Medication histories were screened to exclude medications - including phenytoin and phenobarbital, known to contain propylene glycol or 1,2-propanediol-that generate a doublet resonance on ${ }^{1} \mathrm{H}-\mathrm{MR}$ spectroscopy at $1.1 \mathrm{ppm}$, which could potentially confound results.

MR spectroscopy data were acquired axially for all subjects on a $3 \mathrm{~T}$ Trio magnet (Siemens, Erlangen, Germany) with a multivoxel point-resolved spectroscopy sequence acquisition centered on the basal ganglia $(16 \times 16$ matrix with a VOI of $8 \times 8$, TE $=$ $288, \mathrm{TR}=1700$, slice thickness $=15 \mathrm{~mm}, \mathrm{NEX}=2$, time of acquisition $=5$ minutes 16 seconds). Slab location was assigned in a standardized fashion per our clinical MR spectroscopy protocol, with generally equivalent anatomic sampling across patients (see Fig 1 for a sample slab position). Twenty-two of $23 \mathrm{MR}$ spectroscopy examinations were performed with sedation, using either nitrous oxide/sevoflurane or propofol for induction and sevoflurane or propofol for maintenance.

Data were processed using LCModel (Version 6.3; http:// www.lcmodel.com/) to generate phase and frequency corrected spectra. ${ }^{11}$ Spectral arrays were masked to exclude the outer row within the VOI, as well as any individual spectrum having $\mathrm{N}$-acetylaspartate signal Cramér-Rao lower bounds of $\geq 15$, a typical criterion for spectral quality. The remaining voxels were summed to create a single composite spectrum for each subject.

The composite spectra were reprocessed in LCModel with the addition of a simulated doublet centered at $1.2 \mathrm{ppm}$, correspond- 
ing to the dominant $\mathrm{BHB}$ resonance produced by its methyl group $\left(-\mathrm{CH}_{3}\right)$ (see Fig 1 for a sample fitted summed spectrum). The additional low-amplitude multiplet resonances of BHB at 2.3-2.4 ppm and 4.1 ppm were not modeled.

Results were expressed as a ratio of tissue BHB concentration to commonly assessed brain metabolite concentrations, including NAA, creatine/phosphocreatine (Cr), choline-containing compounds (Cho), and lactate. Derived metabolite ratios were correlated to serum $\mathrm{BHB}$ concentrations, corrected for age in months at the time of MR spectroscopy acquisition.

Analyses were also performed on 3 exploratory datasets (one with a restricted sampling lag threshold of 14 days $[n=13]$, a second with an extended sampling lag threshold of 120 days $[n=$ 31], and a third including all examinations regardless of sampling lag $[n=49])$ to estimate the effect of sampling lag on the strength of correlations.

\begin{tabular}{|c|c|c|c|c|}
\hline Patient & Age (yr) & Epilepsy Syndrome $e^{21-23}$ & Seizure Type & Medications \\
\hline 1 & 1 & EE (Unclassified) & $T$ & LEV \\
\hline 2 & 2 & Metabolic (Mito) & TS & ZNS/LTG/LEV \\
\hline 2 & 7 & Metabolic (Mito) & TS/GTC & ZNS/LTG/LEV/RFM \\
\hline 2 & 8 & Metabolic (Mito) & TS/MY/GTC & ZNS/LTG/LEV/RFM \\
\hline 3 & 9 & MAE & $\mathrm{MY} / \mathrm{AT} / \mathrm{GTC}$ & VPA/CLB/RFM \\
\hline 4 & 7 & Metabolic (Mito) & $\mathrm{MY} / \mathrm{RFL}$ & TPM \\
\hline 4 & 10 & Metabolic (Mito) & $\mathrm{MY} / \mathrm{RFL}$ & TPM/ZNS \\
\hline 5 & 7 & $\mathrm{EE}(\mathrm{MCD})$ & $\mathrm{T} / \mathrm{AT} / \mathrm{TS}$ & VGB/LTG \\
\hline 6 & 2 & Structural (MCD) & Focal & ZNS/TPM \\
\hline 7 & 1 & Structural (Aicardi) & ES & VGB \\
\hline 8 & 6 & Genetic (SCNIA) & MY/AT & LEV/ETX/CLN \\
\hline 9 & 6 & Metabolic (Mito) & MY/AT & LEV/LTG/ETX/CLN \\
\hline 10 & 6 & MAE & $\mathrm{T} / \mathrm{MY} / \mathrm{TS}$ & LTG \\
\hline 11 & 0.5 & Genetic (ATPIA3) & Focal/SE & LEV \\
\hline 11 & 1 & Genetic (ATPIA3) & SUB/MY & LTG/CLB \\
\hline 12 & 6 & EE (unclassified) & $\mathrm{AT} / \mathrm{T} / \mathrm{GTC}$ & CLB \\
\hline 13 & 1 & EE (unclassified) & ES & VGB \\
\hline 14 & 2 & Metabolic (Mito) & ES & TPM \\
\hline 15 & 4 & $\mathrm{EE}(\mathrm{LGS})$ & $\mathrm{AT} / \mathrm{T} / \mathrm{AA}$ & LTG/LEV/ZNS \\
\hline 16 & 1 & EE (Unclassified) & ES & ZNS/LEV \\
\hline 17 & 5 & Genetic (DEAF l) & T/AT/GTC & LEV \\
\hline 18 & 1 & Metabolic (Mito) & ES & ZNS \\
\hline
\end{tabular}

Note:- “Epilepsy Syndrome”: EE indicates epileptic encephalopathy; Mito, primary mitochondrial disease; MAE, myoclonic astatic epilepsy; MCD, malformation of cortical development; LGS, Lennox-Gastaut Syndrome; "Seizure Type": T, tonic; TS, tonic spasms; GTC, generalized tonic-clonic; MY, myoclonic; AT, atonic; RFL, reflexive seizure; ES, epileptic spasms; SE, status epilepticus; AA, atypical absence; SUB, subclinical seizures; "Medications": LEV, levetiracetam; ZNS, zonisamide; LTG, lamotrigine; RFM, rufinamide; CLB, clobazam; TPM, topiramate; VGB, vigabatrin; ETX, ethosuximide; CLN, clonazepam; VPA, valproic acid.

\section{Statistical Analysis}

Descriptive analyses were performed for all variables, with age at MR spectroscopy in months covaried for parametric Spearman correlations. Significance was set at $P<.05$, with the primary time point of interest being a lag of $\leq 30$ days. Other lag intervals were analyzed to explore the strength of the correlation, with reported values not corrected for multiple comparisons and/or autocorrelation owing to overlapping samples with decreasing lags. Analyses were performed in SPSS 19 (IBM, Armonk, New York).

\section{RESULTS}

Clinical data for the 18 included patients are summarized in Table 1. Nineteen datasets were obtained from patients on the KD, and 4 were from patients treated with the MAD. Mean blood serum BHB concentrations were $35.2 \pm 25.9 \mathrm{mg} / \mathrm{dL}$ (range, $0.2-81.3 \mathrm{mg} / \mathrm{dL}$ ). The mean lag time between blood sampling and MR spectroscopy was $11.1 \pm 9.7$ days (range, $0-28$ days).

Included spectra $(n=23)$ were acquired at a mean patient age of $54.0 \pm$ 37.3 months (range, 1-129 months). In terms of spectral fit parameters, the mean SNR was $25.7 \pm 6.3$ (range, 12 36 ), and the mean full width at half maximum was $0.04 \pm 0.01$ (range, 0.02 0.06 ), with mean NAA Cramér-Rao lower bounds of $1.9 \pm 0.6$ (range, $1-3$ ).

Using age as a covariate, we calculated correlation coefficients between blood levels and brain metabolites. They are reported in Table 2. BHB blood levels were strongly correlated to brain BHB levels (calculated correlation coefficient for blood BHB-BHB/NAA was $R=0.81$ with $P<.001$; calculated correlation coefficient for blood $\mathrm{BHB}-$ BHB/Cr was $R=0.80$ with $P<.001$ ) and inversely but more weakly related to NAA levels, regardless of the ratio denominator (calculated correlation coefficient for blood BHB-NAA/Cr was $R=$ -0.55 with $P<.01$; calculated correlation coefficient for blood BHB-NAACho was $R=-.44$ with $P<.04)$. No

Table 2: Partial correlations of blood BHB concentration $(\mathrm{mg} / \mathrm{dL})$ and brain metabolite ratios derived from ' $\mathrm{H}$-MRS, using patient age in months as a covariate

\begin{tabular}{|c|c|c|c|c|c|c|c|c|c|}
\hline Group & $\mathrm{BHB} / \mathrm{Cr}$ & $\mathrm{BHB} / \mathrm{Cho}$ & $\mathrm{BHB} / \mathrm{NAA}$ & $\mathrm{BHB} / \mathrm{Lac}$ & Cho/Cr & $\mathrm{NAA} / \mathrm{Cr}$ & NAA/Cho & Lac/NAA & $\mathrm{Lac} / \mathrm{Cr}$ \\
\hline \multicolumn{10}{|c|}{ Lag $\leq 14 \mathrm{~d}(n=13)$} \\
\hline$R$ & .871 & .898 & .886 & .507 & -.287 & -.645 & -.540 & .376 & .253 \\
\hline Significance & .000 & .000 & .000 & .092 & .367 & .024 & .070 & .228 & .428 \\
\hline \multicolumn{10}{|c|}{$\operatorname{Lag} \leq 30 \mathrm{~d}(n=23)$} \\
\hline$R$ & .803 & .814 & .807 & .538 & -.165 & -.553 & -.443 & .421 & .334 \\
\hline Significance & .000 & .000 & .000 & .010 & .463 & .008 & .039 & .051 & .128 \\
\hline \multicolumn{10}{|c|}{ Lag $\leq 120 \mathrm{~d}(n=31)$} \\
\hline$R$ & .790 & .802 & .781 & .484 & -.312 & -.658 & -.515 & .523 & .423 \\
\hline Significance & .000 & .000 & .000 & .008 & .099 & .000 & .004 & .004 & .022 \\
\hline \multicolumn{10}{|l|}{ All $(N=49)$} \\
\hline$R$ & .579 & .588 & .584 & .393 & -.170 & -.387 & -.312 & .290 & .162 \\
\hline Significance & .000 & .000 & .000 & .006 & .254 & .007 & .033 & .048 & .278 \\
\hline
\end{tabular}

Note:- Lac indicates lactate. 

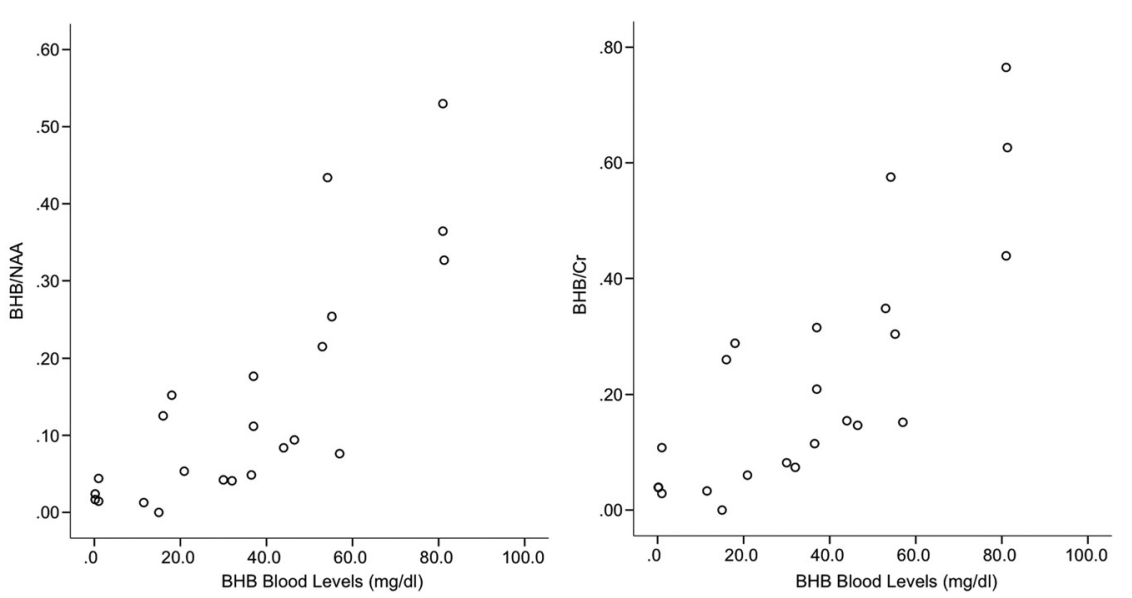

FIG 2. Scatterplot of blood serum BHB concentrations (milligrams/deciliter) versus brain BHB/ $\mathrm{NAA}$ and $\mathrm{BHB} / \mathrm{Cr}$ ratios demonstrates the strong associations between measures.
$\mathrm{BHB}$ ratios to serum $\mathrm{BHB}$ levels, though we had a relative paucity of children on the MAD. That the specific diet type did not appear to alter the observed correlation suggests that brain $\mathrm{BHB}$ levels may be correlated with serum BHB concentrations independent of the dietary mechanism used to achieve ketosis, be it increased fat ratio or carbohydrate restriction. Further evaluation of a cohort with larger subgroups of diet type would be useful to confirm this observation.

We demonstrated an inverse relationship between blood levels of $\mathrm{BHB}$ and brain NAA ratios, independent of patient age. This is in contrast to a prior animal study in rats with diabetic ketoacidosis, which found no significant dif- strong concordance with lactate was demonstrated. BHB/NAA and $\mathrm{BHB} / \mathrm{Cr}$ plots versus blood $\mathrm{BHB}$ concentration for individual examinations are shown in Fig 2.

Results were calculated for the exploratory datasets, also reported in Table 2. For the dataset with a threshold lag of 14 days, the calculated correlation coefficient for blood BHB-BHB/NAA was $R=0.89$ with $P<.001$. For the dataset with a threshold lag of 120 days, the calculated correlation coefficient for blood BHB$\mathrm{BHB} / \mathrm{NAA}$ was $R=0.781$ with $P<.001$. When the entire cohort was included, blood BHB-BHB/NAA demonstrated an $R=0.584$ with $P<.001$.

\section{DISCUSSION}

Prior reports have demonstrated ketone bodies by ${ }^{1} \mathrm{H}-\mathrm{MR}$ spectroscopy in small numbers of mainly adult patients during prolonged fasting, ${ }^{4,12}$ diabetes, ${ }^{13,14}$ and BHB supplementation ${ }^{15}$ and in patients treated with the KD. ${ }^{9,10,12,16,17}$ To date, elevated BHB levels have been anecdotally reported in a only 4 children using ${ }^{1} \mathrm{H}$-MR spectroscopy. ${ }^{9,10}$ In our cohort, we demonstrated routine detection of $\mathrm{BHB}$ in pediatric patients treated with the $\mathrm{KD}$ or a modified KD using long-TE multivoxel ${ }^{1} \mathrm{H}-\mathrm{MR}$ spectroscopy performed at $3 \mathrm{~T}$.

High concordance between steady-state brain parenchymal $\mathrm{BHB}$ ratios and serum $\mathrm{BHB}$ levels was observed using a lag threshold between blood sampling and MR spectroscopy of 30 days (Fig 2). The strength of this correlation progressively increased as the lag threshold between blood BHB sampling and MR spectroscopy decreased, suggesting that the concordance reflects a true physiologic relationship. Results persisted when the lag threshold increased to 120 days, with a similar but weaker relationship demonstrated when the entire cohort was included, implying that $\mathrm{BHB}$ ratios are relatively stable with time in patients on the KD. These findings suggest that ${ }^{1} \mathrm{H}-\mathrm{MR}$ spectroscopy might be an effective tool for noninvasive monitoring of ketosis in children on the $\mathrm{KD}$, particularly when performed in conjunction with routine follow-up MR imaging.

In our cohort, seizure response dictated varying ratios of fat to carbohydrates and protein on the KD. Some patients required the MAD for compliance or tolerability concerns. The specific formulation of the diet did not appear to alter the correlation of brain ference in NAA/Cr ratios between study and control animals using ${ }^{1} \mathrm{H}$-MR spectroscopy performed at 7T with a TE of $132 \mathrm{~ms} .{ }^{18}$ Our finding may reflect in vivo changes in amino acid handling that occur with prolonged ketosis in the KD. ${ }^{7,19}$ Increased acetylcoenzyme A as a by-product of ketone body metabolism in the brain drives increased tricarboxylic acid cycling, resulting in increased conversion of cytosolic aspartate to glutamate. Aspartate serves as the precursor molecule for NAA, while glutamate is converted to the inhibitory neurotransmitter GABA. Thus, the observed decrease in NAA may serve as a marker for an increase in GABA production, one of the proposed mechanisms of seizure control in the KD. Further studies using an MR spectroscopy acquisition specifically tailored to interrogate brain GABA concentrations would be instructive, with specific evaluation of the relationship between serum $\mathrm{BHB}$ concentrations and both brain GABA ratios and the degree of seizure control.

Consistent with past studies using infusion samples, lactate ratios did not show significant correlation to BHB serum levels. ${ }^{4}$ This finding has the caveat that anything other than elevated lactate exists near the noise floor, which can preclude accurate association assessment. In 5/18 patients, mitochondrial cytopathy was the etiology of their drug-resistant epilepsy, contributing further uncertainty to ascribing lactate levels to dietary effects.

Of note, the ${ }^{1} \mathrm{H}-\mathrm{MR}$ spectroscopy spectra included in our analyses were acquired with a TE of $288 \mathrm{~ms}$, optimized for the detection of lactate. J-coupling for BHB is $6.3 \mathrm{~Hz}$, suggesting that TEs of $160 \mathrm{~ms}(1 / \mathrm{J})$ or $320 \mathrm{~ms}(2 / \mathrm{J})$ would be more appropriate for optimization of BHB detection. ${ }^{10}$ Furthermore, this TE selection limits the evaluation of additional ketosis-related metabolites, including acetone and acetoacetate. Both metabolites have generally lower brain concentrations ${ }^{4}$ and shorter T2 relaxation times ${ }^{10}$ than BHB. Future work including short-echo data would be helpful to assess these metabolites.

An additional limitation of our methodology was inclusion of gray and white matter and CSF within the VOI. Tissue segmentation was not attempted, and the differential contributions of different tissue types to the composite spectra were not quantified. The presence of visually identifiable BHB peaks in parenchymal voxels suggests that the ketogenic diet metabolites are broadly 
distributed across tissue types, consistent with the work of Pan et al. ${ }^{4}$ Future work would benefit from specific analysis of the contributions of different tissue types to the overall summed spectrum.

Our described methodology, preprocessing and summing a multivoxel dataset to increase the signal-to-noise ratio, allowed routine detection of BHB in brain parenchyma. Further work using same-day blood sampling; integrated tissue-type segmentation using an approach like localization by adiabatic selective refocusing, ${ }^{20}$ which minimizes chemical shift artifacts; absolute metabolite quantification; as well as evaluation of longitudinal changes will be helpful to extend and refine these results.

\section{CONCLUSIONS}

We demonstrated routine detection of a brain parenchymal BHB using conventional ${ }^{1} \mathrm{H}-\mathrm{MR}$ spectroscopy in the brains of pediatric patients with epilepsy treated with the KD. We further demonstrated a strong correlation between brain tissue BHB ratios and serum BHB concentrations, apparently independent of specific diet formulation. These findings suggest that ${ }^{1} \mathrm{H}-\mathrm{MR}$ spectroscopy may be a useful noninvasive method of interrogating brain tissue BHB levels in patients on the KD or a modified KD protocol. The observation of an inverse correlation between brain tissue NAA levels and serum BHB levels may reflect altered amino acid handling induced by the KD, consistent with one of the proposed antiepileptic mechanisms of the KD.

\section{REFERENCES}

1. Wheless JW. History of the ketogenic diet. Epilepsia 2008;49(Suppl 8):3-5 CrossRef Medline

2. Brodie MJ. Antiepileptic drug therapy the story so far. Seizure 2010; 19:650-55 CrossRef Medline

3. Kim JA, Yoon JR, Lee EJ, et al. Efficacy of the classic ketogenic and the modified Atkins diets in refractory childhood epilepsy. Epilepsia 2016;57:51-58 CrossRef Medline

4. Pan JW, Rothman TL, Behar KL, et al. Human brain beta-hydroxybutyrate and lactate increase in fasting-induced ketosis. J Cereb Blood Flow Metab 2000;20:1502-07 CrossRef Medline

5. Pan JW, Telang FW, Lee JH, et al. Measurement of beta-hydroxybutyrate in acute hyperketonemia in human brain. J Neurochem 2001; 79:539-44 Medline

6. Owen OE, Morgan AP, Kemp HG, et al. Brain metabolism during fasting. J Clin Invest 1967;46:1589-95 CrossRef Medline

7. Bough KJ, Rho JM. Anticonvulsant mechanisms of the ketogenic diet. Epilepsia 2007;48:43-58 Medline

8. van Delft R, Lambrechts D, Verschuure P, et al. Blood beta-hydroxybutyrate correlates better with seizure reduction due to ketogenic diet than do ketones in the urine. Seizure 2010;19:36-39 CrossRef Medline
9. Novotny EJ, Rothman DL. Observation of cerebral ketone bodies by proton nuclear magnetic resonance spectroscopy [abstract]. Ann Neurol 1996;40:285 CrossRef

10. Cecil KM, Mulkey SB, Ou X, et al. Brain ketones detected by proton magnetic resonance spectroscopy in an infant with Ohtahara syndrome treated with ketogenic diet. Pediatr Radiol 2015;45:133-37 CrossRef Medline

11. Provencher SW. Estimation of metabolite concentrations from localized in vivo proton NMR spectra. Magn Reson Med 1993;30: 672-79 CrossRef Medline

12. Schubert F, Mekle R, Ittermann B, et al. Ketone bodies and glucose in human brain during ketogenic diet and fasting. In: Proceedings of the Annual Meeting and Exhibition of the International Society for Magnetic Resonance in Imaging, Toronto, Ontario, Canada. May 30-June 5,2015

13. Kreis R, Ross BD. Cerebral metabolic disturbances in patients with subacute and chronic diabetes mellitus: detection with proton MR spectroscopy. Radiology 1992;184:123-30 CrossRef Medline

14. Wootton-Gorges SL, Buonocore MH, Kuppermann N, et al. Detection of cerebral $\{$ beta\}-hydroxybutyrate, acetoacetate, and lactate on proton MR spectroscopy in children with diabetic ketoacidosis. AJNR Am J Neuroradiol 2005;26:1286-91 Medline

15. Plecko B, Stoeckler-Ipsiroglu S, Schober E, et al. Oral beta-hydroxybutyrate supplementation in two patients with hyperinsulinemic hypoglycemia: monitoring of beta-hydroxybutyrate levels in blood and cerebrospinal fluid, and in the brain by in vivo magnetic resonance spectroscopy. Pediatr Res 2002;52:301-06 Medline

16. Seymour KJ, Bluml S, Sutherling J, et al. Identification of cerebral acetone by $1 \mathrm{H}-\mathrm{MRS}$ in patients with epilepsy controlled by ketogenic diet. MAGMA 1999;8:33-42 Medline

17. Artzi M, Liberman G, Vaisman N, et al. Changes in cerebral metabolism during ketogenic diet in patients with primary brain tumors: 1H-MRS study. J Neurooncol 2017;132:267-75 CrossRef Medline

18. Glaser N, Ngo C, Anderson S, et al. Effects of hyperglycemia and effects of ketosis on cerebral perfusion, cerebral water distribution, and cerebral metabolism. Diabetes 2012;61:1831-37 CrossRef Medline

19. Yudkoff M, Daikhin Y, Nissim I, et al. Brain amino acid metabolism and ketosis. J Neurosci Res 2001;66:272-81 CrossRef Medline

20. Scheenen TW, Klomp DW, Wijnen JP, et al. Short echo time $\mathbf{1 H}-$ MRSI of the human brain at 3T with minimal chemical shift displacement errors using adiabatic refocusing pulses. Magn Reson Med 2008;59:1-6 CrossRef Medline

21. Berg AT, Berkovic SF, Brodie MJ, et al. Revised terminology and concepts for organization of seizures and epilepsies: report of the ILAE Commission on Classification and Terminology, 2005-2009. Epilepsia 2010;51:676-85 CrossRef Medline

22. Scheffer IE, Berkovic S, Capovilla G, et al. ILAE classification of the epilepsies: position paper of the ILAE Commission for Classification and Terminology. Epilepsia 2017;58:512-21 CrossRef Medline

23. Bernier FP, Boneh A, Dennett X, et al. Diagnostic criteria for respiratory chain disorders in adults and children. Neurology 2002;59: 1406-11 CrossRef Medline 